\title{
Point-to-Point Communication on the Moon *
}

\author{
L. E. Vogler
}

(Received July 19, 1962)

\begin{abstract}
This paper presents a preliminary study of point-to-point communication systems on the surface of the moon. Ground wave propagation is assumed over a lunar model consisting of a smooth sphere of homogeneous material in free space and attenuation curves are presented for a wide range of electromagnetic ground constants. The communication system is described in terms of the power required at the input terminals of the transmitting antenna in order to obtain a given signal-to-noise ratio at the receiver. Discussions of antenna considerations and noise effects are presented and an example is given of a system composed of a Beverage wave antenna transmitting towards a vertical electric dipole. For ground conductivities on the order of $10^{-4}$ to $10^{-3} \mathrm{mhos} / \mathrm{m}$ this example indicates an optimum frequency lying in the LF band and a communication range out to somewhat beyond 100 kilometers, depending on the type of service desired.
\end{abstract}

\section{Introduction}

The problem of determining the requirements necessary for adequate point-to-point communication systems on the surface of the moon is, at the present time, complicated by a lack of information concerning lunar composition and environment. The presence or absence of an ionosphere, the values to assume for conductivity, permeability, and permittivity of the lunar surface material, and the possibility of layering below the surface are factors affecting any consideration of communication requirements. The effect of extra-lunar noise sources on signal reception can only be estimated using information gained from earth-based measurements. Until such time as actual experiments on the moon can be undertaken to provide the needed information, lunar communication studies can give only a general indication of what may be expected in the way of power requirements and antenna types.

The lunar model assumed for the purposes of the present paper is a smooth sphere of radius $r_{0}(=1738$ $\mathrm{km}$ ) consisting of a homogeneous material characterized by a relative dielectric constant $\boldsymbol{\epsilon}_{r}$, conductivity $\sigma$ in mhos per meter, and magnetic permeability equal to that of free space. The mks system of units is used throughout this paper. The effects of any atmosphere or ionosphere surrounding the model are neglected. The primary mechanism of propagation is considered to be the ground wave, the solution of which is well known and may be expressed by the Van der Pol-Bremmer residue series for diffracted waves around a smooth homogeneous sphere [Bremmer, 1949]. Because of the difficulties inherent in the construction of any complex antenna structures on the moon, low antenna heights are assumed and, consequently, height gain effects are considered negligible.

\footnotetext{
*Sponsored by the Jet Propulsion Laboratory, Pasadena, Calif.
}

\section{System Loss}

The concept of system loss [Norton, 1953, 1959; Wait, 1959] will be used to describe the effects of the various system parameters. System loss $L$ is defined as the ratio, expressed in decibels, ${ }^{1}$ of the available power $p_{t}$ supplied at the input terminals of the transmitting antenna to the available power $p_{\tau}$ delivered to the receiver if there were no circuit losses other than those associated with antenna terminal resistances:

$$
L \equiv 10 \log \left(p_{t} / p_{r}\right)=P_{t}-P_{r},
$$

where a conjugate matching of impedances at the receiver end is assumed and only linear systems are considered. The system loss may be divided further into a free space or inverse distance component which would be the loss expected between two isotropic antennas situated in free space, an attenuation $A_{t}$ relative to free space which accounts for the effects on propagation of the intervening terrain, and two transmitting and receiving antenna components, $L_{t}-G_{t}$ and $L_{r}-G_{r}$, which describe the effects of the particular antennas used. Thus, (1) may be written as

$$
\begin{aligned}
P_{t}=L+P_{r}=10 & \log \left(4 \pi d_{0} / \lambda\right)^{2} \\
& +A_{t}+\left(L_{t}-G_{t}\right)+\left(L_{r}-G_{r}\right)+P_{r},
\end{aligned}
$$

where $d_{0}$ is the arc distance separating the twc antennas measured around the sphere and $\lambda$ is the free space wavelength expressed in the same units as $d_{0} . \quad G_{t, r}$ is the transmitting or receiving antenna gain relative to an isotropic antenna, and $L_{t, r}$ is an antenna loss arising from its proximity to the ground.

${ }^{1}$ In this paper capital letters are used to designate the decibel equivalent of the corresponding lower case letters, e.g., $P_{t}=10 \log _{10} p_{t}$. 
Notice that in order to calculate the available power from the output terminal of the transmitter, any transmitter transmission line loss should be added to the right side of (2). However, with reasonable care the effect of this loss may be made negligible, at least at the lower frequencies.

Equation (2) provides a formula to find the transmitted power necessary to obtain a given received power. However, the power received consists not only of the desired signal but also of radio noises arising both from within and without the receiving system. If $r$ denotes the desired minimum signalto-noise power ratio which will provide a given grade of reception as measured at the receiver predetection output, and an effective noise figure $f$ is defined as the ratio of the signal-to-a reference Johnson-noise power ratio which is available from a loss-free receiving antenna to the receiver predetection output signal-to-noise ratio, then the received power may be expressed [Norton, 1959] as

$$
p_{r}=r f k_{B} t_{0} b / l_{r},
$$

where $k_{B} t_{0} b$ is the reference Johnson-noise power, $k_{B}$ is Boltzmann's constant $\left(=1.38044 \times 10^{-23}\right.$ joules degree), $t_{0}$ is a reference temperature in degrees Kelvin, and $b$ is the effective noise bandwidth in cycles per second. The definition of $f$ in terms of noise figures of the component parts of the receiving network can be shown to be [Barsis, Norton, Rice, and Elder, 1961]

$$
f=f_{e}-1+f_{c t r}
$$

where $f_{e}$ is the "noise figure" of the external noise and $f_{c t r}$ is the noise figure of the antenna circuit, transmission line, and receiver. These are discussed in more detail in section 5 .
Using the above definitions, the transmitted power may now be expressed as

$$
\begin{aligned}
P_{t}=20 \log \left(4 \pi d_{0} / \lambda\right) & +A_{t}-\left(G_{t}+G_{r}\right)+L_{t} \\
& +R+F+B+10 \log \left(k_{B} t_{0}\right) .
\end{aligned}
$$

The following sections will discuss the various components of (5).

\section{Norton Surface Wave Attenuation, $A_{t}$}

Numerical procedures for the calculation of electromagnetic fields diffracted around a smooth sphere have been developed by various authors [Burrows and Gray, 1941; Norton, 1941; Bremmer, 1949]. The ground wave may be expressed as a series of residues which, if the functions of antenna height are equal to unity, depend on the radius of the sphere $r_{0}$, the arc distance $d_{0}$ separating the antennas, the free space wavelength $\lambda$, the relative dielectric constant $\epsilon_{\tau}$ and conductivity $\sigma$ of the ground, and the polarization of the wave. To emphasize the fact that no height gain effects are included in the analysis presented in this paper, the term "Norton surface wave" [Norton, 1941] is used to denote the ground wave with antenna heights set equal to zero. In parametric form the Norton surface wave attenuation relative to an inverse distance field $A_{t}$ is conveniently plotted as a function of three parameters: $K, b^{\circ}$, and $x^{\prime}{ }_{0}$. Definitions of these parameters and their relationship to the residue series are discussed in section 1 of the appendix. In the expressions that follow, a homogeneous ground is assumed. However, it should be mentioned that recent investigations [Wait, 1962] have shown that with a simple extension of the definitions of $K$ and $b^{\circ}$, attenuation curves such as

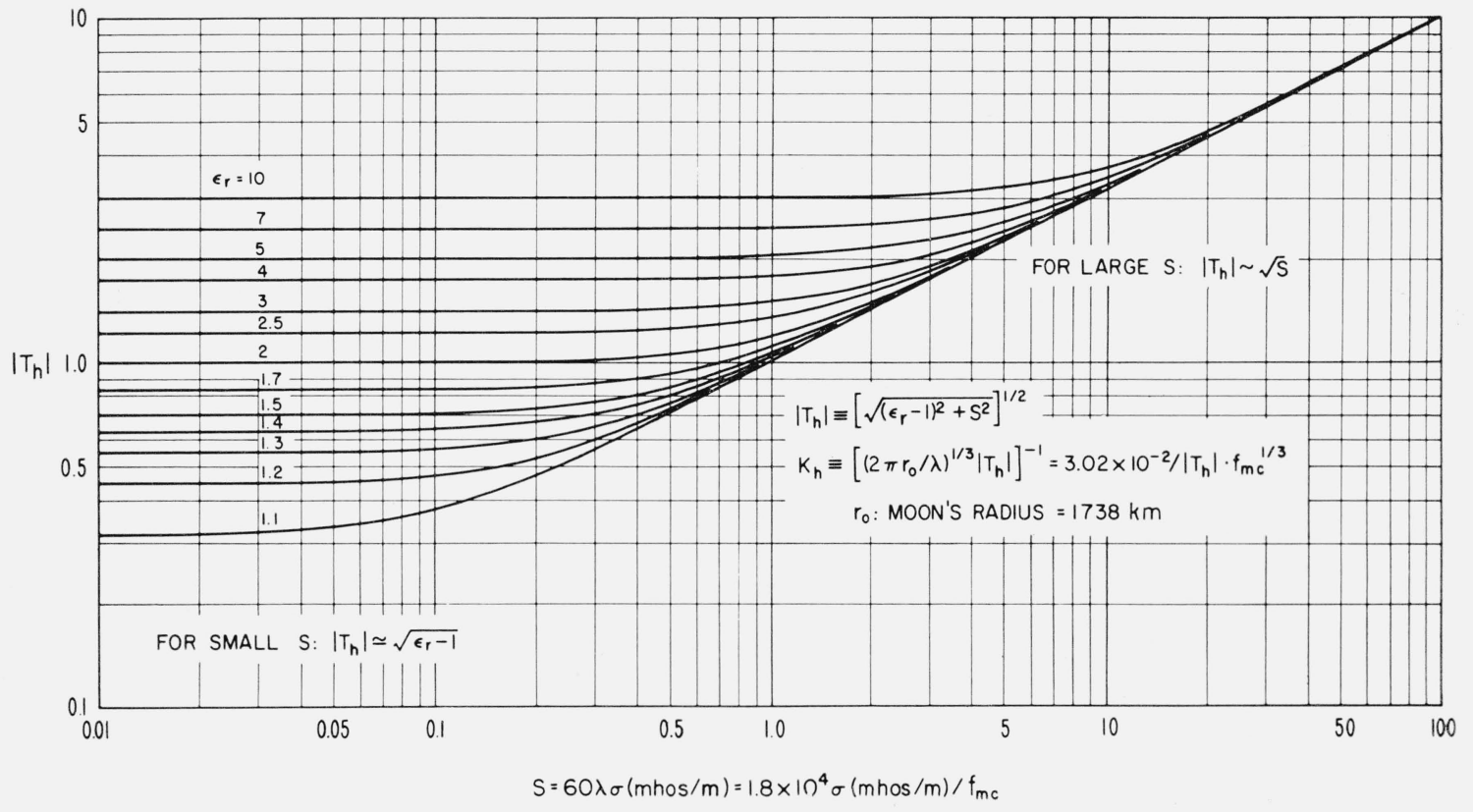

FIGURE 1. The parameter $\left|\mathrm{T}_{\mathrm{h}}\right|$ for horizontal polarization. 
those presented in this paper may be used to calculate propagation effects over stratified material.

For horizontal polarization $\left(K=K_{h}\right)$ and vertical polarization $\left(K=K_{v}\right)$

$$
K_{h}=\left[\left(2 \pi r_{0} / \lambda\right)^{\frac{1}{3}}\left|T_{h}\right|\right]^{-1}, K_{v}=\left[\left(2 \pi r_{0} / \lambda\right)^{\frac{1}{3}}\left|T_{v}\right|\right]^{-1},
$$

where $\left|T_{h}\right|$ and $\left|T_{v}\right|$ are plotted as functions of $\epsilon_{r}$ and $s=60 \lambda \sigma(\mathrm{mhos} / \mathrm{m})$ in figures 1 and 2 . Here $s$ represents the imaginary part of $\mu^{2}$ as defined by Bremmer [1949, page 5], $\lambda$ is the free space wavelength in meters, and $\sigma$ is conductivity in mhos per meter. Values of $\epsilon_{r}>10$ are not shown because present information indicates that the relative dielectric constant of the moon is considerably less than ten. However, $\left|T_{h}\right|$ and $\left|T_{v}\right|$ are easily obtained for other $\epsilon_{r}$ 's since
$\left|T_{h}\right|=\left[\left(\epsilon_{r}-1\right)^{2}+s^{2}\right]^{1 / 4},\left|T_{v}\right|=\left[\sqrt{\left(\epsilon_{r}-1\right)^{2}+s^{2}} /\left(\epsilon_{r}^{2}+s^{2}\right)\right]^{1 / 2}$.

Limiting forms of these functions for small $s$ beyond the range of the graphs are:

$$
\left|T_{h}\right| \simeq \sqrt{\epsilon_{r}-1},\left|T_{v}\right| \simeq \sqrt{\epsilon_{r}-1} / \epsilon_{r}, \quad s \rightarrow 0,
$$

and for large $s$ :

$$
\left|T_{h}\right| \sim \sqrt{ } s,\left|T_{v}\right| \sim 1 / \sqrt{s}, \quad s \rightarrow \infty .
$$

Similar plots of $b_{h}^{\circ}$ and $b_{v}^{\circ}$ (the subscripts $h$ and $v$ again referring to horizontal and vertical polarization respectively) as functions of $\epsilon_{r}$ and $\delta$ are shown in figures 3 and 4 . Expressions for $b_{h}^{\circ}$ and $b_{v}^{\circ}$ are

$$
\begin{aligned}
b_{h}^{\circ}=180^{\circ}-\tan ^{-1}\left[\left(\epsilon_{r}-1\right) / s\right], b_{v}^{\circ}= & 2 \tan ^{-1}\left[\epsilon_{r} / s\right] \\
& -\tan ^{-1}\left[\left(\epsilon_{r}-1\right) / s\right] .
\end{aligned}
$$

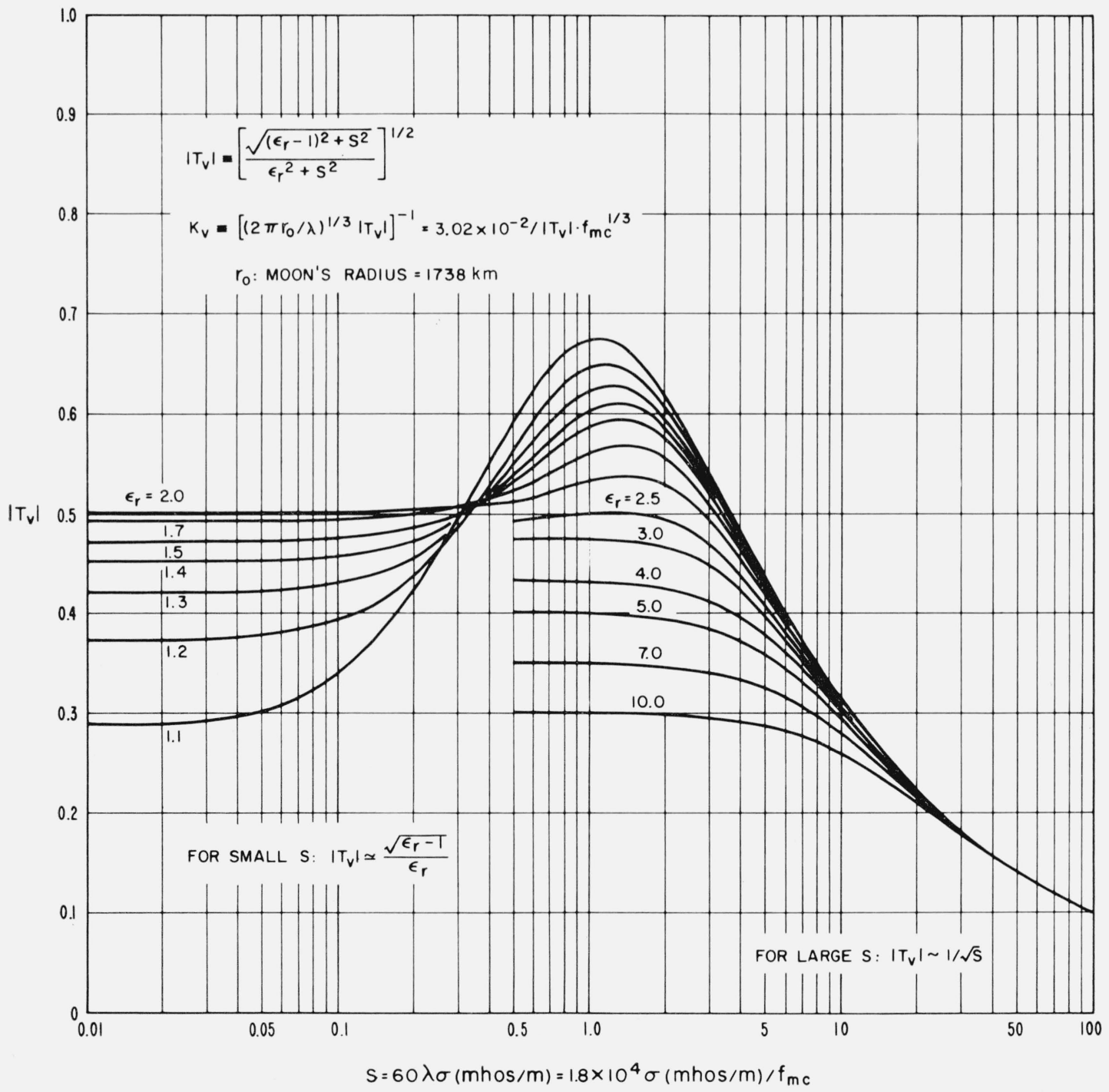

Figure 2. The parameter $\left|\mathrm{T}_{\mathrm{v}}\right|$ for vertical polarization. 


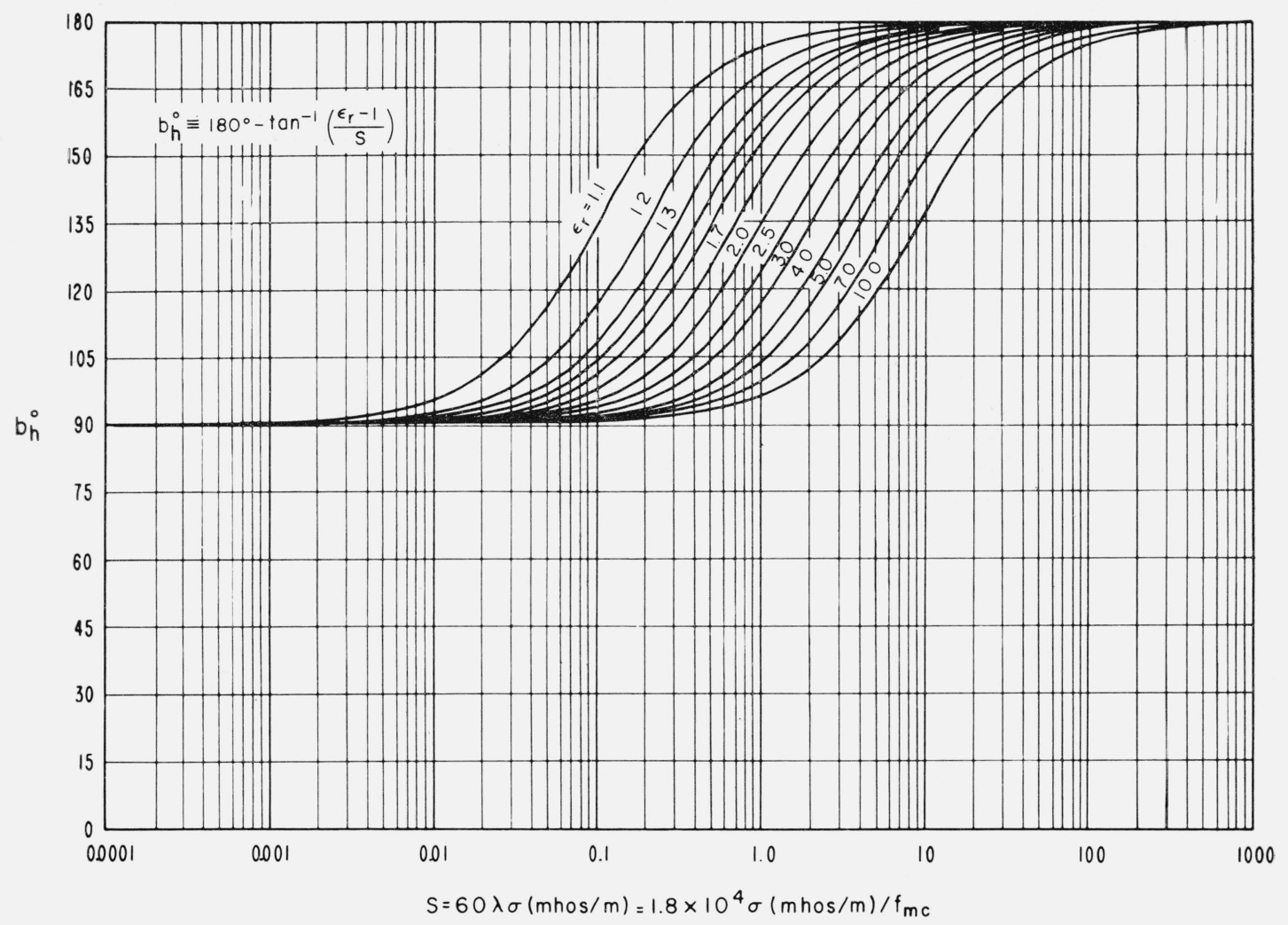

FiguRE 3. The parameter $\mathrm{b}_{\mathrm{h}}^{\circ}$ for horizontal polarization.

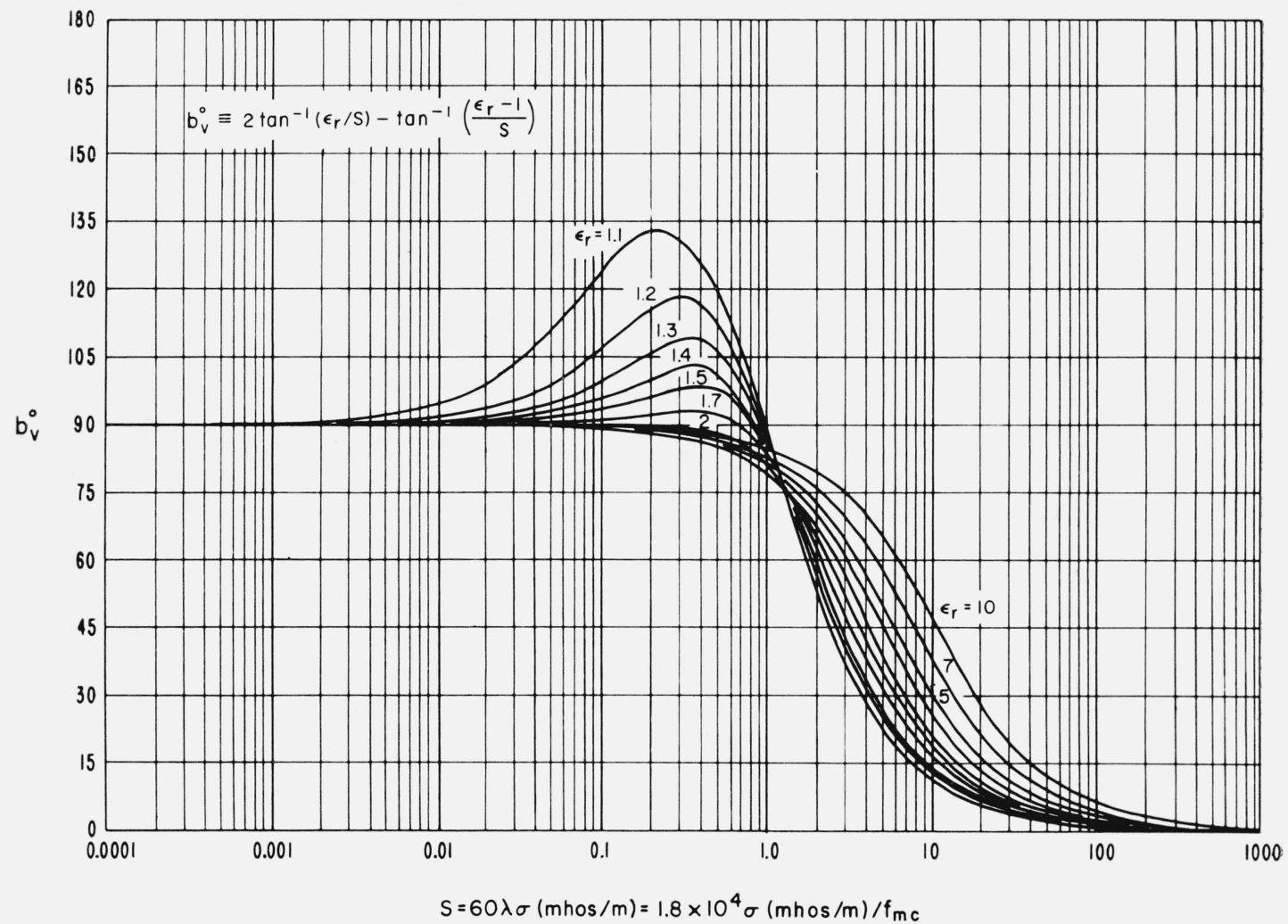

FiguRE 4. The parameter $\mathrm{b}_{\mathrm{v}}^{\circ}$ for vertical polarization. 
The Norton surface wave attenuation $A_{t}$ is graphed in figures 5 through 8 versus the distance parameter $x_{0}^{\prime}$ where

$$
x_{0}^{\prime}=f_{\mathrm{mo}}^{\frac{1}{3}} d_{0}(k m),
$$

with $f_{\mathrm{mc}}$ denoting the frequency in megacycles per second and $d_{0}(\mathrm{~km})$ the distance measured in kilometers. It should be noticed that in general $A_{z}$ does not vary much with $b^{\circ}$ and linear interpolation may be used for values of $b^{\circ}$ other than those shown. Also it can be seen that for $K>10, A_{t}$ approaches a limiting function which is plotted as the top curve on each graph.

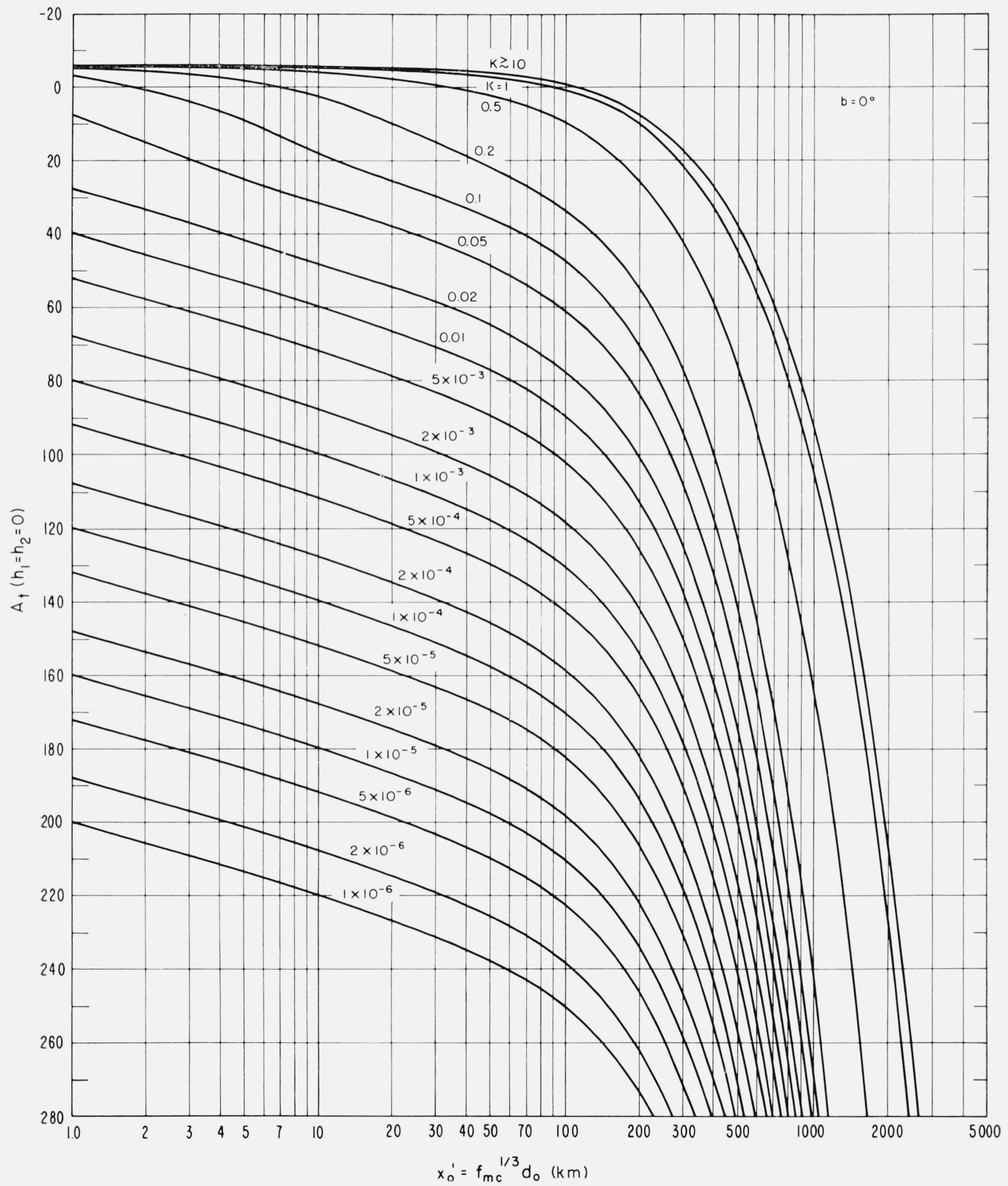

FiguRE 5. Norton surface wave attenuation, $\mathrm{A}_{\mathrm{t}}, \mathrm{b}=0^{\circ}$. 
Equations (2) and (5) are strictly applicable only for the case of antennas separated a sufficient distance apart $\left(d_{0}>>\lambda\right)$ such that the magnitude of their mutual impedance is small compared to the self resistances of the antennas [Norton, 1959]. Because of this restriction $A_{t}$ is not shown in the figures for values of $x_{0}^{\prime}<1$. As $d_{0}$ (or $x_{0}^{\prime}$ ) goes to zero, $A_{t}$ approaches $20 \log (1 / 2)=-6.02$, which would correspond to the surface wave field intensity expected between short vertical electric dipoles situated near each other on a perfectly conducting plane.

It is apparent from figures 5 through 8 that the attenuation is less for the larger values of $K$, these values corresponding to vertically polarized waves at the lower frequencies. For example, with a lunar

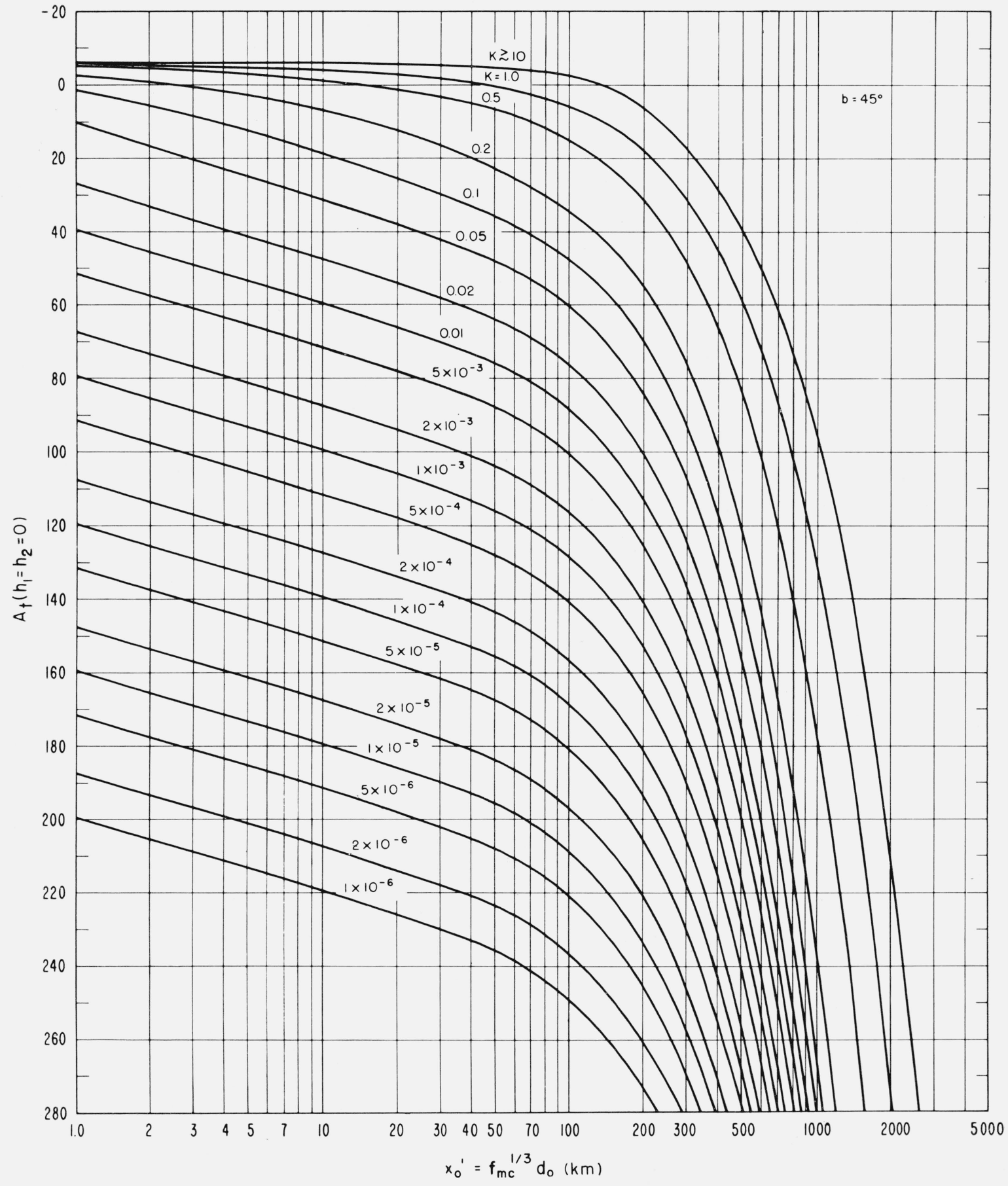

Figure 6. Norton surface wave attenuation, $\mathrm{A}_{\mathrm{t}}, \mathrm{b}=45^{\circ}$. 
ground conductivity of, say, $\sigma=4 \times 10^{-4} \mathrm{mhos} / \mathrm{m}$, and for frequencies less than or equal to about 1 $\mathrm{Mc} / \mathrm{s}$, it is seen from (7b) that $K_{v} \simeq 0.081 f_{\mathrm{me}}^{-5 / 6}$. In this case the attenuation is less than $50 \mathrm{db}$ out to distances of $100 \mathrm{~km}$. On the other hand, with horizontal polarization, $K_{h}$ is usually much less than unity for all frequencies and the attenuation is greater.

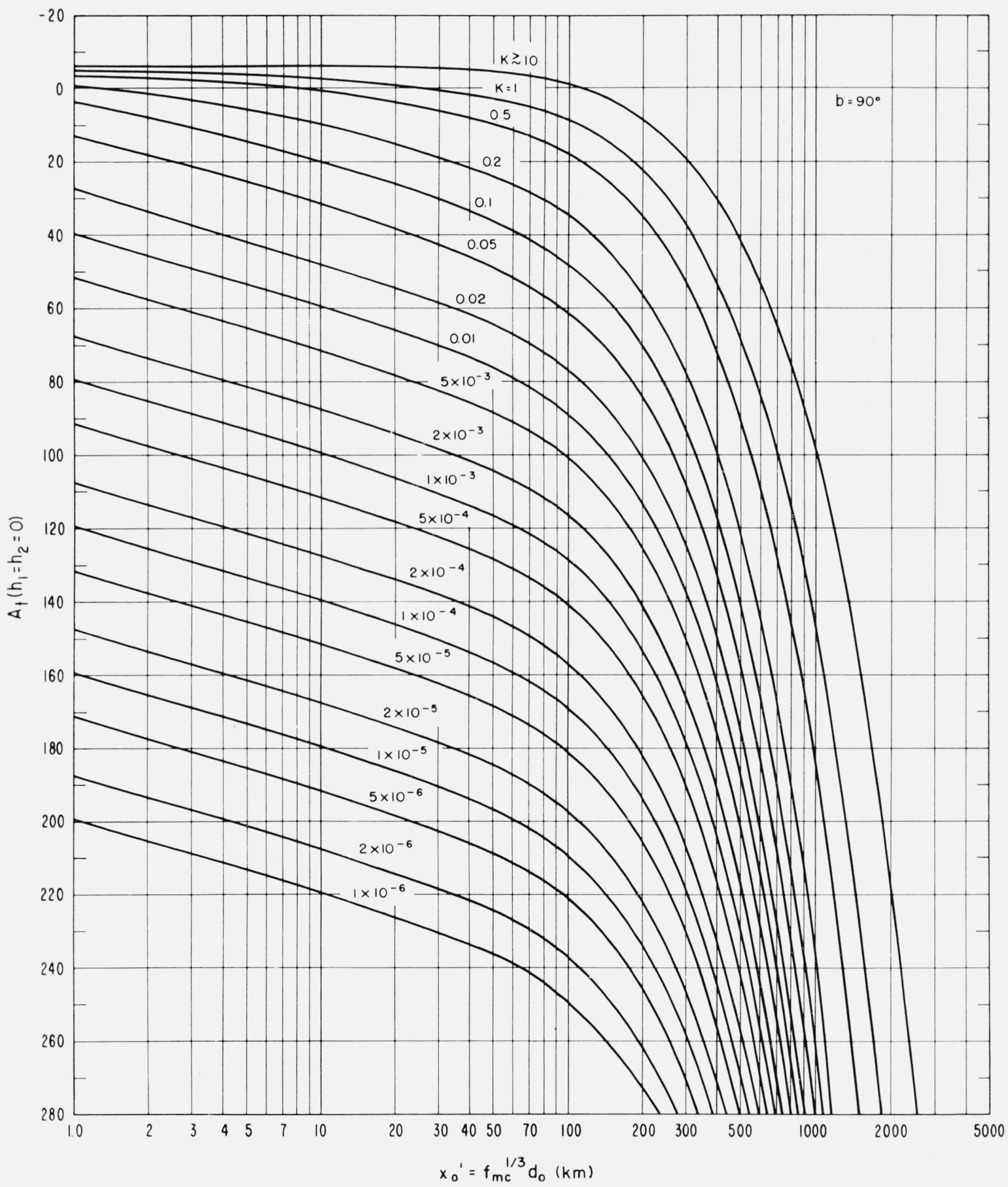

Figure 7. Norton surface wave attenuation, $\mathrm{A}_{\mathrm{t}}, \mathrm{b}=90^{\circ}$. 


\section{Antenna Effects and the Wave Antenna}

The terms $L_{t, r}$ and $G_{t, r}$ (the subscript $t, r$ referring to either transmitting or receiving antenna) in (2) describe the effects of the particular transmitting and receiving antennas used in the communication system under consideration. $L_{t, r}$ is defined as the ratio expressed in decibels of, the terminal resistance of the antenna to its free space radiation resistance. Thus, as the height of the antenna above the surface is increased, the ratio of the resistances approaches unity and $L_{t}$ or $L_{r}$ effectively become zero. For heights near the surface the antenna terminal resistance is a function of the electromagnetic ground constants, $\epsilon_{r}$ and $\sigma$, and for poorly conducting grounds $\mathrm{L}_{t, r}$ may become very large [Wait, 1953]. $G_{t, r}$ denotes the free space gain of an optimally

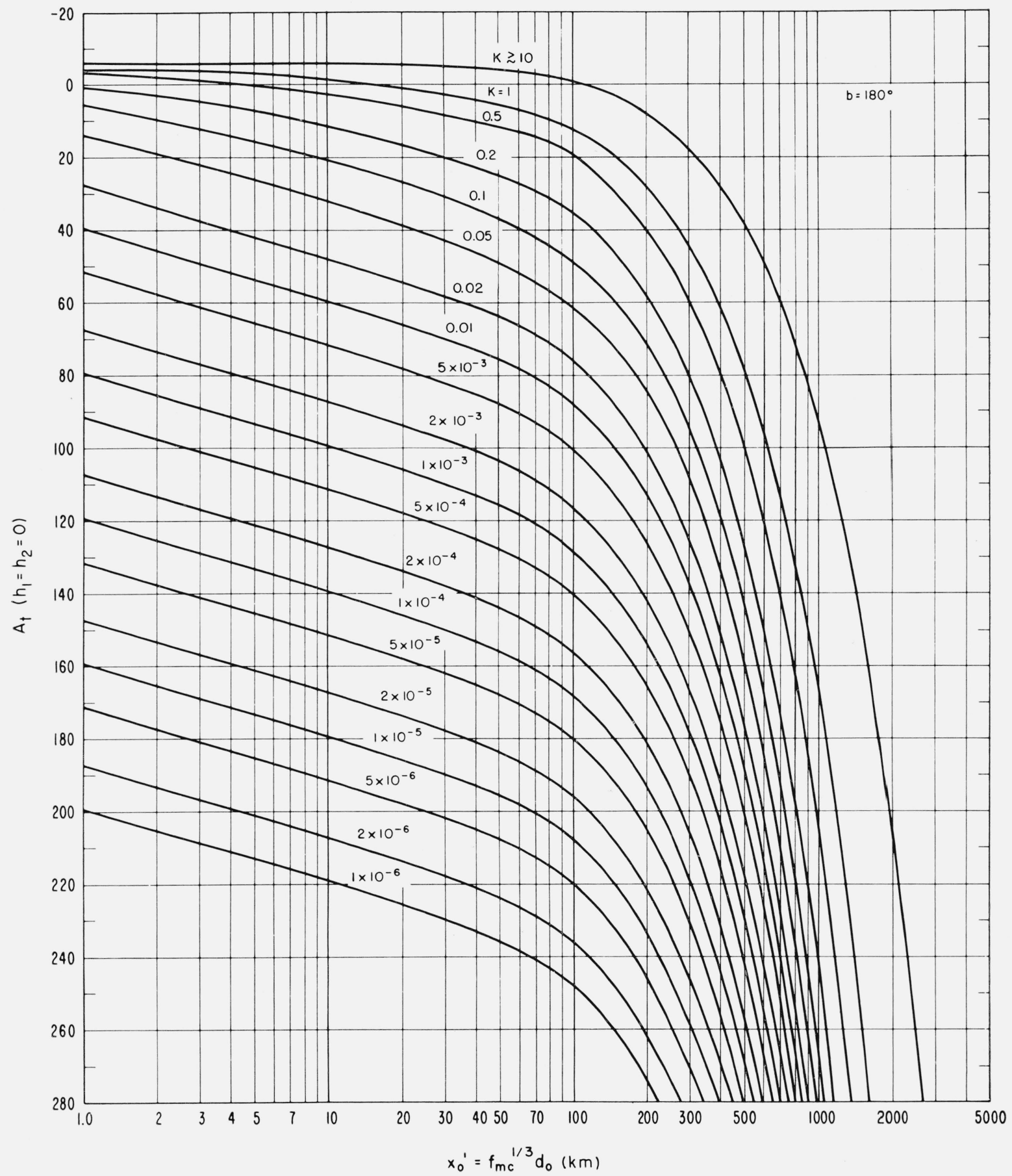

FiguRE 8. Norton surface wave attenuation, $\mathrm{A}_{\mathrm{t}}, \mathrm{b}=180^{\circ}$. 
oriented loss-less antenna above an isotropic antenna, e.g., $G_{t}=G_{r}=10 \log (3 / 2)=1.76$ for electric current elements or elementary dipoles and $G_{t}=G_{r}=2.15$ for half-wave antennas.

Although the separation of antenna effects into a free space gain and a ground proximity loss is somewhat arbitrary, especially in the case of surface wave propagation, it is possible to reduce the effect of the ground and at least approach a free space condition for low antennas through the use of an appropriate ground screen. Discussions of antenna characteristics and their modifications by ground screens will be found in Schelkunoff and Friis [1952] and papers by Wait and Surtees [1954] and by Wait [1956]. The rest of this section will be devoted to a discussion of the Beverage wave antenna [Beverage, Rice, and Kellogg, 1923] and dipole-type antennas.

The wave antenna in its simplest form consists of a long horizontal wire situated a short height above the ground and terminated at one end through its characteristic impedance. It is a unidirectional antenna with the maximum gain in the direction of the antenna axis and toward the terminated end. Wait [1954] has shown that the vertical electric field component of a wave antenna is proportional to a complex factor $T_{v}$, termed the "wave tilt" and defined by

$$
T_{v}=\left[\left(\epsilon_{r}-1\right)-i s\right]^{\frac{1}{2}} /\left(\epsilon_{r}-i s\right),
$$

and a function $S^{\prime}$ which depends on wavelength, electromagnetic ground constants, antenna length and height, and an angle $\phi$ measuring the direction in which the antenna is pointing. $S^{\prime}$, obtained by integrating the contributions of all the elements along the antenna, is given by

$$
S^{\prime}=\frac{1-\exp [-(\Gamma-i \beta \cos \phi) l]}{(\Gamma-i \beta \cos \phi) l}
$$

where $\beta=2 \pi / \lambda, l$ is the antenna length, and $\Gamma$ is the propagation constant of the wire which for large antenna heights approaches the propagation constant of plane waves in free space, $i \beta$. In terms of the wave antenna power gain [Martin and Wickizer, $1949]$ referred to an isotropic antenna, $\left(p_{w a} / p_{\text {iso }}\right)$, this becomes

$$
\left(p_{w a} / p_{i s o}\right)=(\beta l \cos \phi)^{2}\left|S^{\prime}\right|^{2}\left|T_{v}\right|^{2}\left(120 R_{0} /\left|Z_{0}\right|^{2}\right),
$$

where $Z_{0}=R_{0}+i X_{0}$ is the characteristic impedance of the wave antenna. The derivation of $(12)$ is discussed in the appendix, section 2. The factor $\left|T_{v}\right|$ is shown in figure 2 and $\left|S^{\prime}\right|$ is graphed in figure 9 as a function of the two parameters $(\alpha l)$ and $(\beta l)(m-\cos \phi)$, with the propagation constant $\Gamma$ defined as

$$
\Gamma \equiv \alpha+i \beta m,
$$

where $\alpha$ and $m$ are real. Thus when the antenna height $h$ is sufficiently large, $\alpha=0$ and $m=1$. Atlower heights an approximate expression for the values of $\alpha$ and $m$ has been given by Carson [1926]:

$$
\alpha / \beta \simeq m-1 \simeq\left[2^{3 / 2}(\beta h) \sqrt{\epsilon_{r}^{2}+s^{2}} \ln \left(4 h / d_{a}\right)\right]^{-1},
$$

where $d_{a}$ is the diameter of the antenna wire. For an antenna actually lying on the ground, $\Gamma$ may be expressed as [Coleman, 1950]

$$
\begin{aligned}
\Gamma=\alpha+i \beta m=\beta & \sqrt{m^{2}-\frac{1}{2}\left(\boldsymbol{\epsilon}_{r}+1\right)} \\
& +\frac{i \beta}{2}\left[\sqrt{\left(\boldsymbol{\epsilon}_{r}+1\right)^{2}+s^{2}}+\left(\boldsymbol{\epsilon}_{r}+1\right)\right]^{\frac{1}{2}} .
\end{aligned}
$$

It should be noticed from (15) that the propagation constant of a wave antenna lying on the ground may approach its free space value if the relative

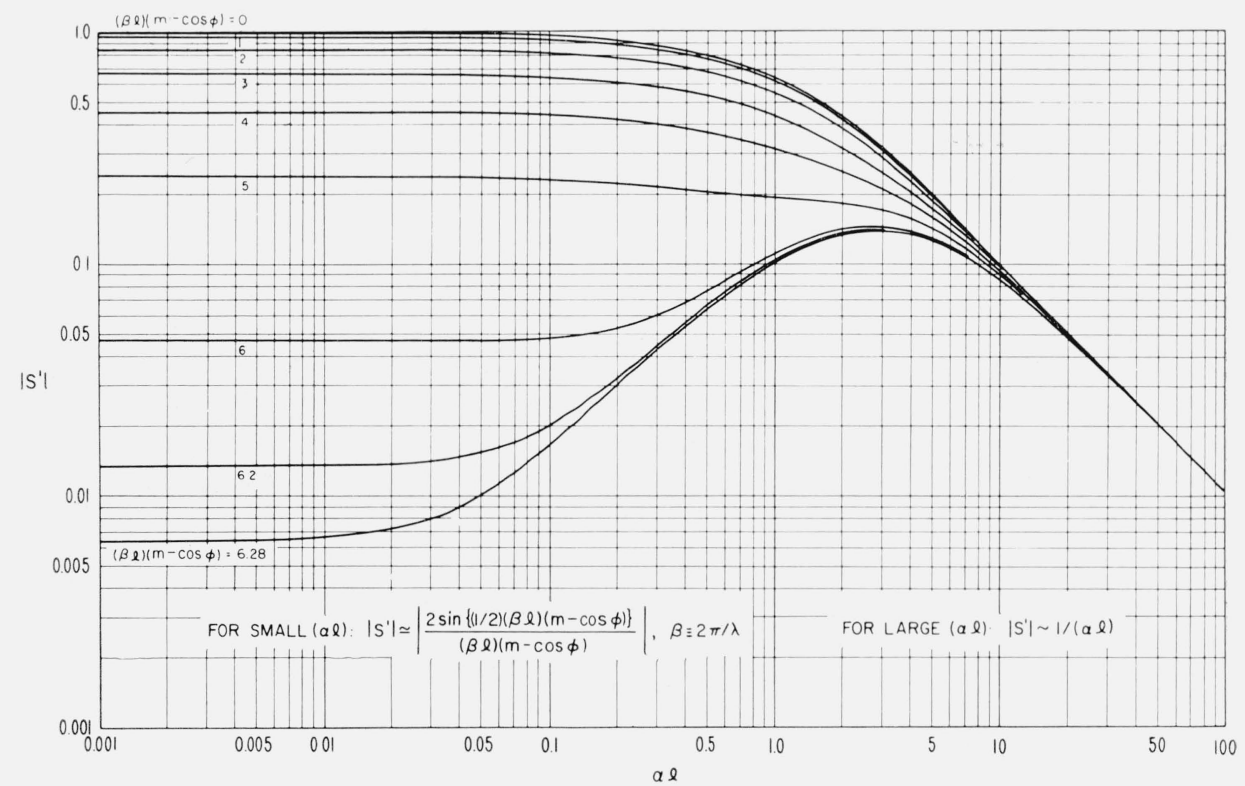

Figure 9. Pattern factor $\left|\mathbf{S}^{\prime}\right|$ for a horizontal traveling wave antenna of length 1. 
dielectric constant is very near unity and the conductivity is extremely small. These conditions appear to hold for lunar surface material. An example of the wave antenna pattern as a function of the direction angle $\phi$ is shown in figure 10 for several values of the parameter $\beta l=2 \pi(l / \lambda)$ and for $m=1, \alpha=0$. It is seen that the antenna is highly directional in the forward direction $(\phi=0)$; other values of $m$ and $\alpha$ modify the pattern somewhat but the general shape is the same.

A modification of the wave antenna which is also discussed in the paper by Wait [1954] is one in which the horizontal wire of length $l=2 b$ is center-fed and the ends of the antenna are not terminated with a load impedance. Assuming a current distribution along the wire of

$$
I(y)=I_{0}[\sinh (\Gamma b)]^{-1} \sinh \{\Gamma(b-|y|)\},
$$

where $I_{0}$ and $y$ are respectively the current at and the distance from the feed point, an integration of the elements over the length of the antenna yields for the antenna pattern $|S|$

$$
\begin{aligned}
|S|=|\beta b \cos \phi| \cdot\left|S_{1}^{\prime}\right| & \\
=\left|x_{3}\right|\left[\frac{2\left\{\cosh x_{1}-\cos \left(x_{2}-x_{3}\right)\right\}}{x_{1}^{2}+\left(x_{2}-x_{3}\right)^{2}}\right. & \\
& \frac{2\left\{\cosh x_{1}-\cos \left(x_{2}+x_{3}\right)\right\}}{x_{1}^{2}+\left(x_{2}+x_{3}\right)^{2}} \\
& \left.\times \frac{x_{1}^{2}+x_{2}^{2}}{\cosh ^{2} x_{1}-\cos ^{2} x_{2}}\right]^{\frac{1}{2}},
\end{aligned}
$$

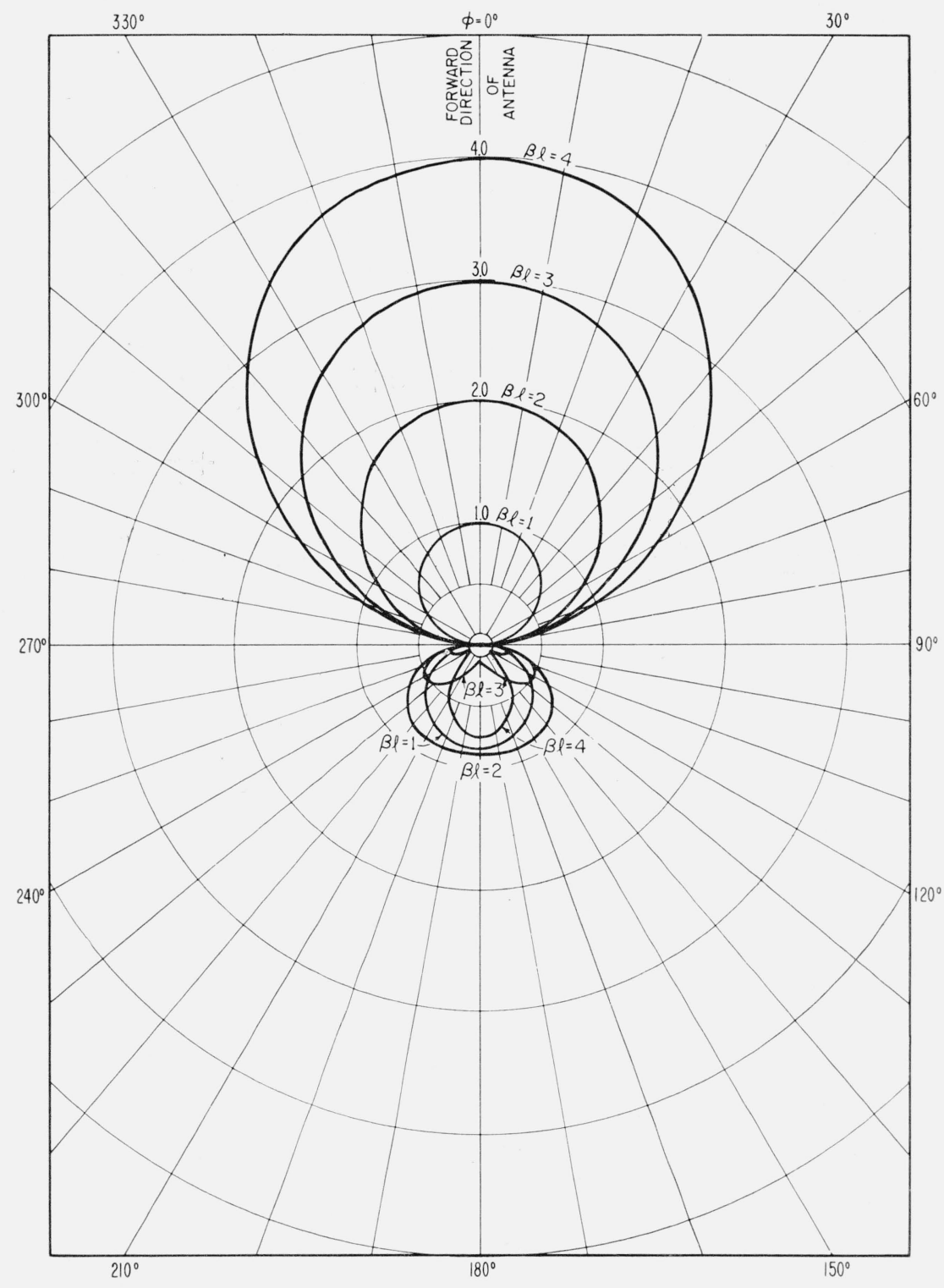

Figure 10. Horizontal traveling wave antenna pattern.

$|\mathrm{S}|=|\beta 1 \cos \phi| \cdot\left|\mathrm{S}^{\prime}\right|$ for $\mathrm{m}=1, \alpha=0$. 
where $x_{1}=\alpha b, x_{2}=\beta b m$, and $x_{3}=\beta b \cos \phi$. The relationship of $|S|$ to the wave antenna power gain is given in section 2 of the appendix. Graphs of (17) for the case $\alpha=0$ are shown in figure 11. Thus, for example, with values of $\alpha \simeq 0$ and $m=2$ (which would correspond to the antenna lying on frozen or dry ground) and an antenna length of $l=2 b=\lambda / 4$, the value of $|S|$ in the forward direction, $\phi=0$, is read from figure 11 as $|S|=0.94$.

The effect of finite ground conductivity is quite pronounced on dipole-type antennas of small heights at the lower frequencies. Considerable power loss occurs as the conductivity approaches zero. Sommerfeld and Renner [1942] have investigated ground proximity effects for the case of horizontal and vertical electric dipoles, and using their expression for the antenna terminal resistance $r$, the quantity $L_{t, r}-G_{t, \tau}$ has been calculated and plotted versus frequency in figure 12 for a vertical electric dipole of height $h=\lambda / 16$ and combinations of ground constants: $\epsilon_{r}=1.1,2.0$, and $\sigma=10^{-3}, 10^{-4}$. As stated at the beginning of this section, $L_{t, r}$ is defined by $L_{t, r}=10 \log \left(r / r_{f}\right)$, where the subscript $f$ refers to free space conditions; $r$, expressed in integral form in the Sommerfeld and Renner paper, was numerically evaluated using an electronic computer and $G_{t, r}$ the free space gain was set equal to 1.76 .

Similar curves for the case of a vertical magnetic dipole-type antenna which is equivalent to a small circular loop with its axis perpendicular to the ground may be obtained from the expression for the radiation resistance derived in a paper by Wait [1953]. As in the case of the vertical electric dipole, the power loss becomes quite large at low antenna heights.

\section{Effective Noise Figure}

The effective noise figure $f$ [Barsis, Norton, Rice, and Elder, 1961] describes the noise of the total receiving system in terms of the noise figures of its component parts:

$$
f=f_{e}-1+f_{c t r}
$$

where [Friis, 1944]

and

$$
f_{c t r}=f_{c}+l_{c}\left(f_{t}-1\right)+l_{c} l_{t}\left(f_{r}-1\right),
$$

$$
f_{c}=1+\left(l_{c}-1\right)\left(t_{c} / t_{0}\right), f_{t}=1+\left(l_{t}-1\right)\left(t_{t} / t_{0}\right) .
$$

$l_{c}$ and $l_{t}$ are the loss factors ${ }^{2}$ (i.e., the ratio of the available input to output powers of the component) of the antenna circuit and transmission line respectively, with $t_{c}$ and $t_{t}$ being their corresponding absolute temperatures; $f_{r}$ designates the receiver noise figure and $t_{0}$ is a reference temperature. Generally speaking, the factors $f_{e}$ and $f_{c}$ will predominate in calculating values of $f$, especially at the lower frequencies [Crichlow, Smith, Morton, and Corliss, 1955]; at high frequencies $f$ depends more on $f_{t}$ and $f_{r}$ which are best obtained by direct measurement. By assuming the transmission line loss to be negligible, we may write (18) as

$f=f_{e}+\left(l_{c}-1\right)\left(f_{\tau}-1+t_{c} / t_{0}\right)+\left(f_{\tau}-1\right), \quad l_{t}=1$.

2 Notice that the symbol $l_{t}$ used in this section is not the same as the transmitting antenna loss $l_{t}$ discussed in section 2 .

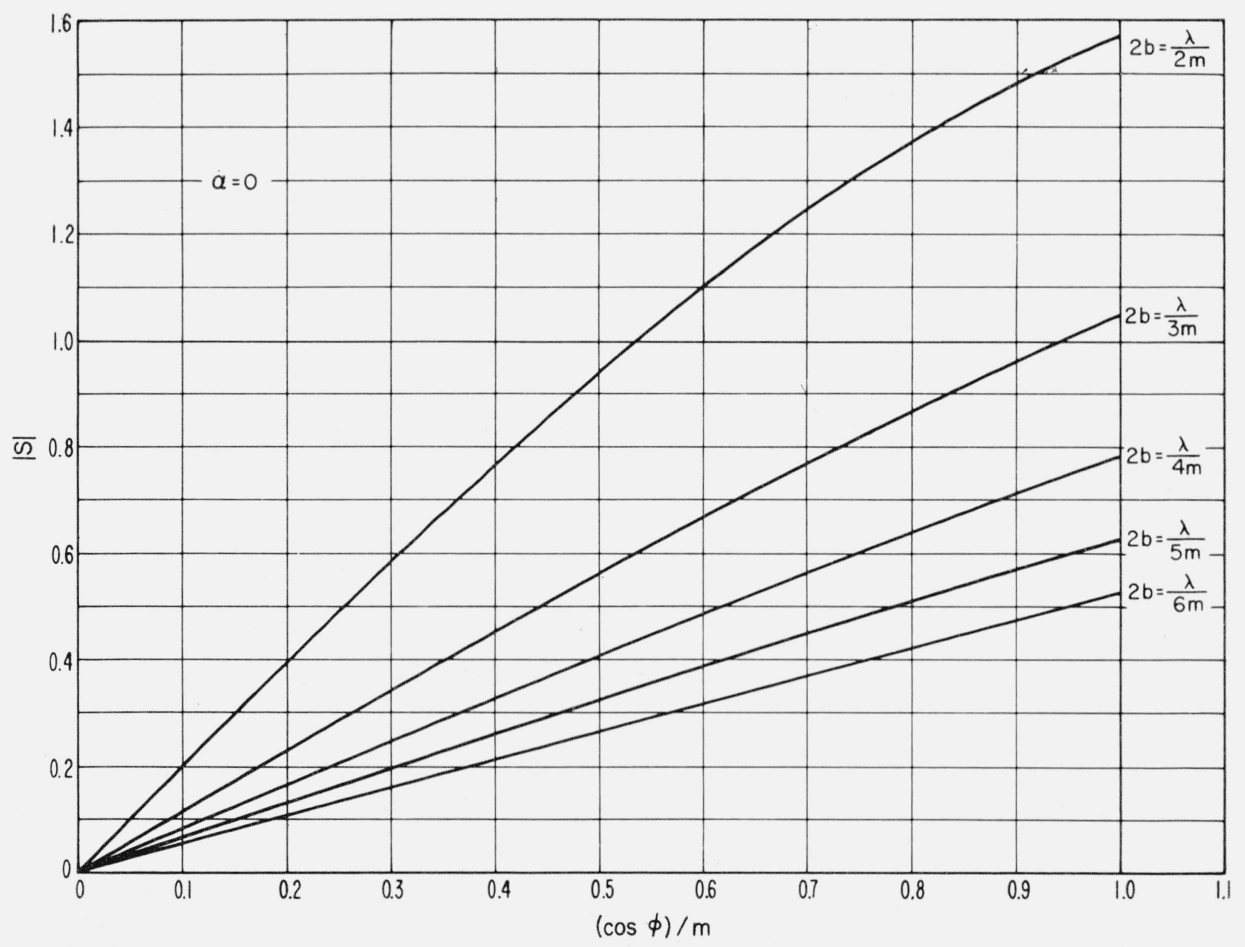

FiguRe 11. $|\mathrm{S}|$ for center-fed wave antenna of length $\mathrm{l}=2 \mathrm{~b}$ with $\alpha=0$. 


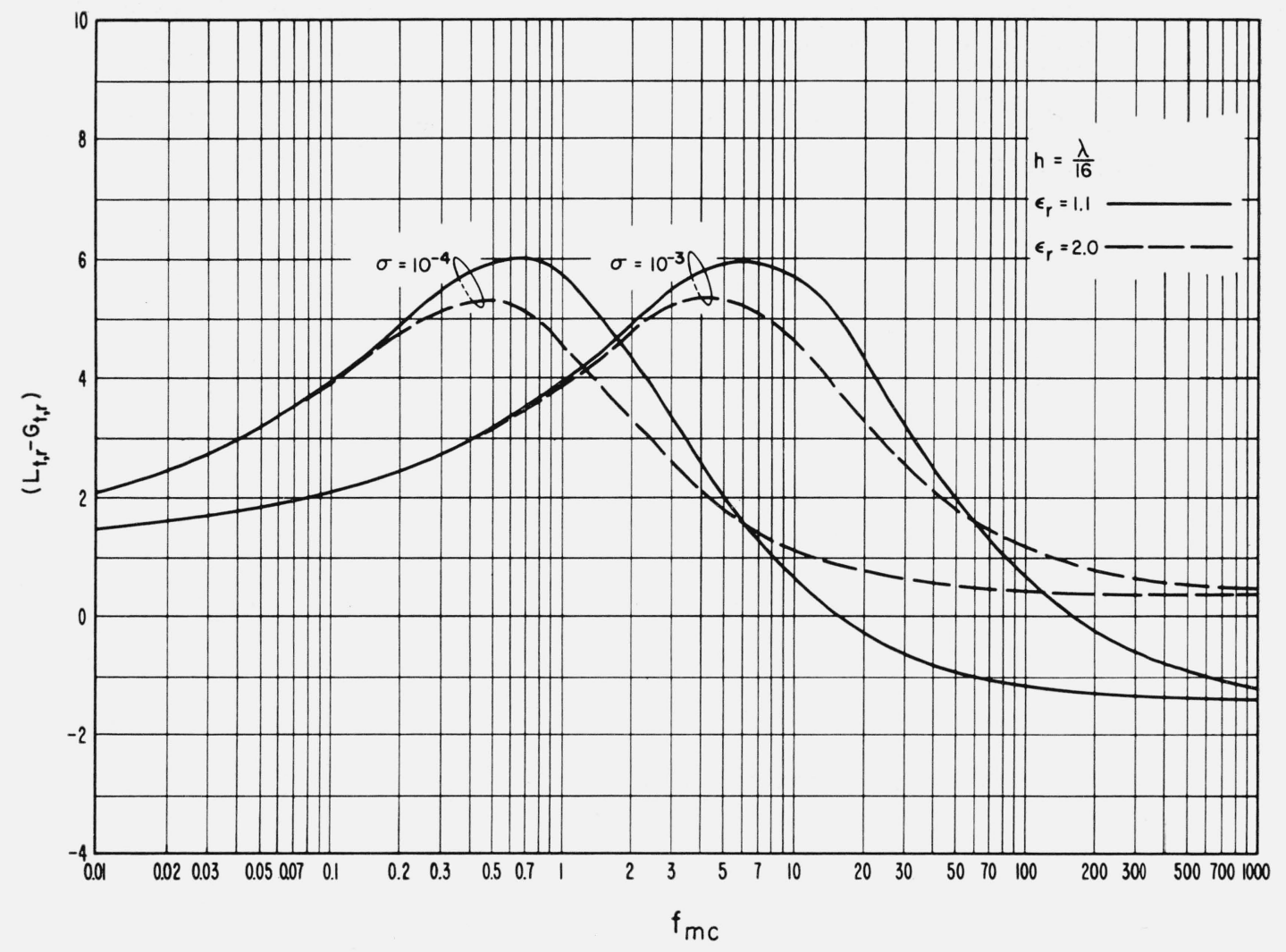

Figure 12. $\left(\mathrm{L}_{\mathrm{t}, \mathrm{r}}-\mathrm{G}_{\mathrm{t}, \mathrm{r}}\right)$ for vertical electric dipole

The effective noise figure now depends on the loss associated with the antenna circuit $l_{c}$, the absolute temperature of the antenna $t_{c}$, the receiver noise figure $f_{r}$, and $f_{e}$, which is a measure of the external noise.

At the present time, of course, and until actual measurements can be undertaken, the amount of external noise received by an antenna located on the moon can be only roughly estimated. It is likely that the predominant source will be galactic; however, consideration also should be given to solar noise and noise arising from the nearby earth. An estimate of galactic noise can be obtained from radio noise maps prepared by Donald $\mathrm{H}$. Menzel of the Harvard College Observatory [Menzel]. These maps also give noise intensities of discrete galactic sources for various frequencies. Using the frequency variation indicated in the text accompanying these maps, $F_{e}=10 \log f_{e}$ (considering galactic noise only) is plotted versus frequency in figure 13 for a reference temperature $t_{0}=288.39^{\circ} \mathrm{K}$. Until data from noise measurements taken above the earth's ionosphere are available, it can be only assumed that the frequency variation is as indicated in the figure for frequencies less than the plasma frequency of about $20 \mathrm{Mc} / \mathrm{s}$. The empirical expression used to plot the galactic noise curve is

$$
\begin{aligned}
& f_{e}=1.585 \times 10^{5} f_{\mathrm{me}}^{-2.3}, \quad f_{\mathrm{mc}} \leq 200 ; \\
& f_{e}=6.467 \times 10^{6} f_{\mathrm{mc}}^{-3}, \quad f_{\mathrm{mc}} \geq 200 .
\end{aligned}
$$

In the case of solar noise and noises from terrestrial sources, it is probable that noticeable effects generally will occur only at the lower frequencies, except during periods of extreme solar activity when considerable noise at all frequencies may be expected [Brown and Lovell, 1957]. Also, of course, some radio noise will be generated by the surface of the moon itself. No attempt is made in the present paper to investigate these aspects of the problem.

The antenna circuit noise figure $f_{c}$, depending as it does on the antenna loss and absolute temperature of the antenna, will vary according to the type of antenna used, the elements of the circuit, and whether the antenna is in direct sunlight or not. Antenna loss is best obtained by direct measurement; however, if measurements are impractical, $l_{c}$ may be approximated by the "ground-proximity" loss $l_{r}$ discussed in section 4 . In fact, for matched conditions of the elements of the receiving antenna circuit, $l_{c}$ and $l_{r}$ are identical. 


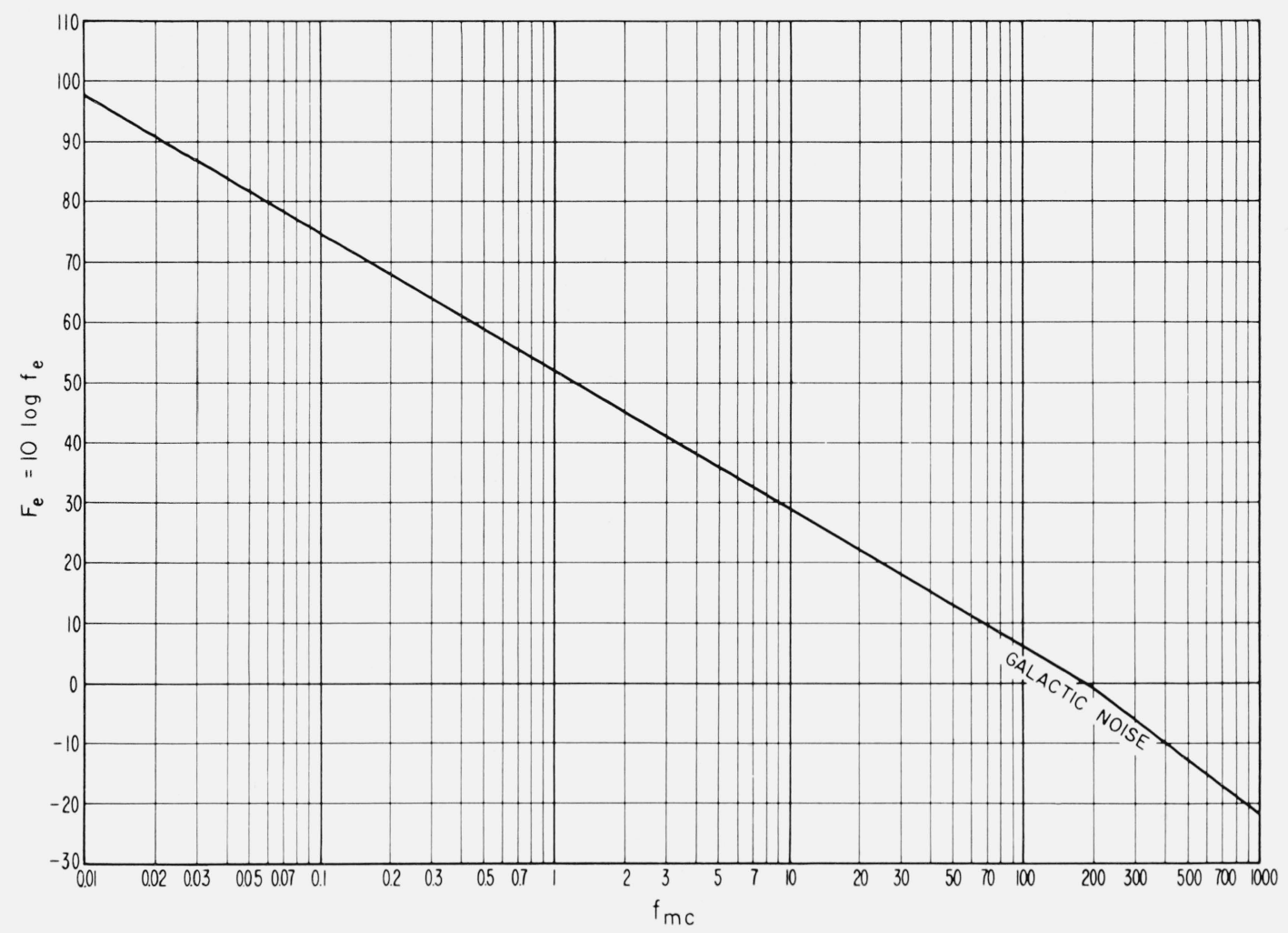

Figure 13. External noise figure, $\mathrm{F}_{\mathrm{e}}$.

\section{Calculation of Required Power}

Attempts have been made recently to deduce the electromagnetic properties of the moon's surface material through the use of radar data [Senior and Siegel, 1960]. Although differences exist concerning: the exact interpretation of the data [Brown, 1960; Daniels, 1961], there is general agreement that the relative permittivity is not far above unity and that the conductivity is quite low. In any case the graphs discussed in the preceding sections are applicable to a wide range of values of $\boldsymbol{\epsilon}_{r}$ and $\sigma$.

Senior and Siegel in the paper cited above estimate the relative permittivity and conductivity of lunar surface material to be $\epsilon_{r}=1.1$ and $\sigma=3.4 \times 10^{-4}$ mhos $/ \mathrm{m}$. Materials constituting the earth's crust have larger values, although some substances such as extremely dry, coarse quartzitic sand are somewhat this order of magnitude. For the purpose of arriving at some estimate of lunar propagation conditions, it will be assumed that $\epsilon_{r}$ ranges from 1.1 to 2.0 and $\sigma$ lies between $10^{-3}$ and $10^{-4} \mathrm{mhos} / \mathrm{m}$.

If we measure the arc distance $d_{0}$ in kilometers and choose a reference temperature $t_{0}=288.39{ }^{\circ} \mathrm{K}$ (as in section 5), the required transmitted power given by (5) may be rewritten as

$$
P_{t}=L_{b}-\left(G_{t}+G_{\tau}\right)+L_{t}+R+F+B-204,
$$

where $L_{b}$ is called the basic transmission loss and is given by

$L_{b}=20 \log \left(4 \pi d_{0} / \lambda\right)+A_{t}=32.45+20 \log d_{0}(\mathrm{~km})$

$$
+20 \log f_{\mathrm{me}}+A_{t} \text {. }
$$

Figure 14 shows curves of $L_{b}$ for vertically polarized waves as a function of frequency and for the distances indicated. Values of $A_{t}$ were obtained from figures 5 through 8 by linear interpolation in both the $K_{v}$ and $b_{v}^{\circ}$ directions, $K_{v}$ being given by figure 2 and eq (6), and $b_{v}^{\circ}$ being read from figure 4 . Notice that the basic transmission loss varies inversely with conductivity at the lower frequencies while at high frequencies, the effect of variation with $\sigma$ becomes negligible.

If we now assume a communication system consisting of, for example, a horizontal traveling wave antenna lying on the moon's surface and transmitting towards a short vertical electric dipole placed some distance away and in an optimum direction from the transmitter $\left(\phi \simeq 0^{\circ}\right)$, we may estimate the power requirements from (21). An estimate of $L_{t}-G_{t}$ may be obtained from (12) (see section 2 of the appendix) by assuming the free space value for the wave antenna characteristic impedance: $\left|Z_{0}\right| \sim R_{0}=120 \pi$. Using figures 2 and $9, L_{t}-G_{t}=-10 \log \left(p_{w a} / p_{\text {is o }}\right)$ was calculated for the case of an antenna length $l=\lambda / 4$ and plotted versus frequency in figure 15 . As before with basic transmission loss, the antenna loss 


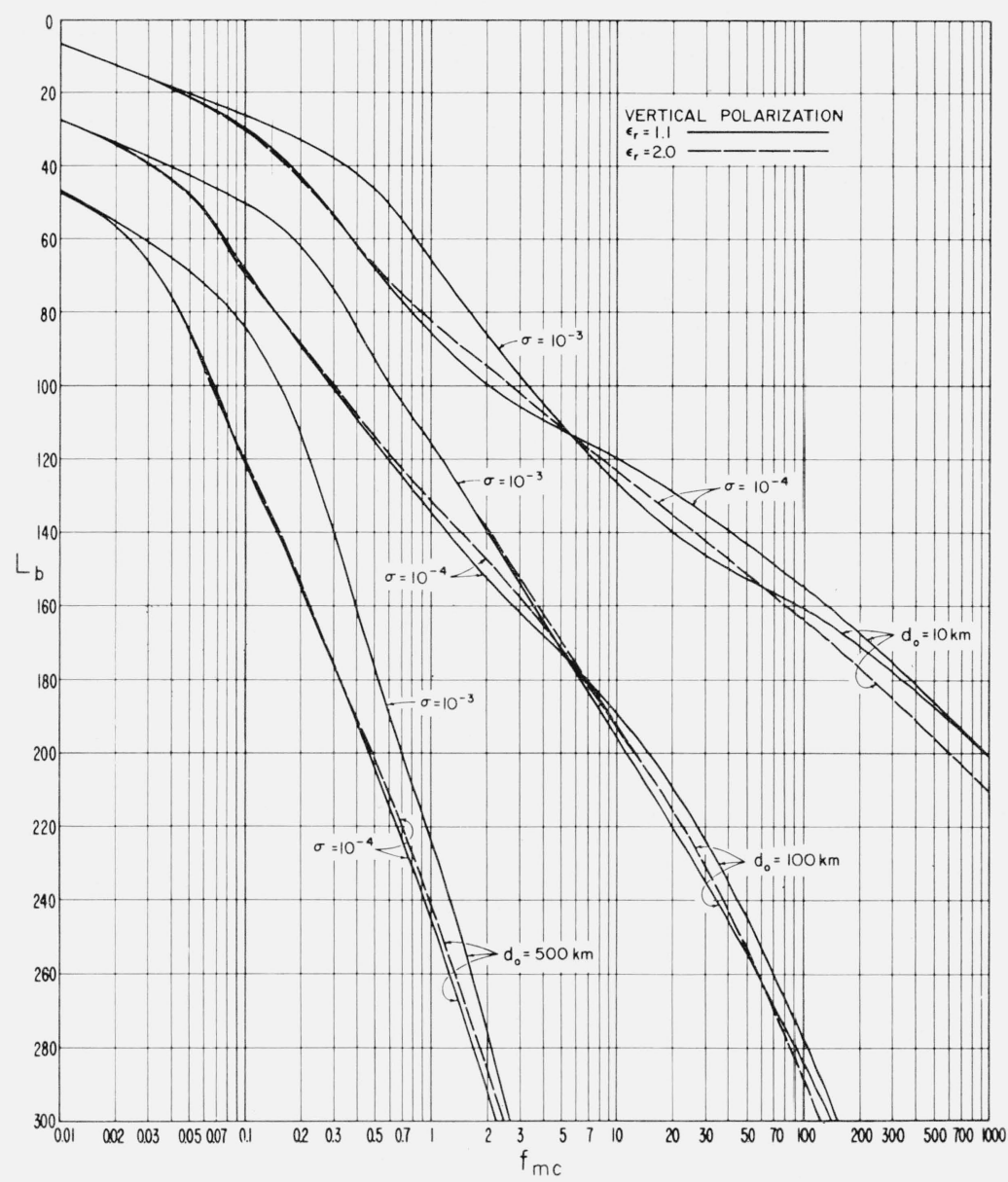

Figure 14. Basic transmission loss for vertically polarized Norton surface waves.

at the lower frequencies varies noticeably with conductivity but not with the relative permittivities assumed; at higher frequencies, the opposite is true.

By comparing figures 12 and 13 , it can be seen that the external noise tends to "blanket out" the effect of the receiving antenna circuit loss. Thus, if we assume the effective noise figure to be a function only of the external noise $f_{e}$ and a receiver noise figure of, say, $f_{r}=4$ :

$$
F=10 \log \left(f_{e}+3\right), l_{t}=l_{c}=1,
$$

the required transmitted power for a given type of service under the above restrictions can be calculated from (21) by settin $G_{r}=1.76$ and making use of figures 14 and 15 , and eq (23). Figure 16 shows values of $P_{t}-(R+B)$ as a function of frequency for antenna separation distances of 10,100 , and $500 \mathrm{~km}$. The wave antenna length of $l=\lambda / 4$ and vertical dipole height of $h=\lambda / 16$ were arbitrarily chosen, the main consideration being the complexity of the physical structures of the antennas. Of course at low frequencies even antennas of these dimensions might be very difficult to construct under working conditions on the moon. Notice that at very short distances propagation will be by line-of-sight rather than surface wave due to the dipole being at a height other than zero. The transmission loss in this case should be calculated by the standard geometrical optics method.

It should be emphasized that this particular combination of antennas is meant merely to illustrate the use of (21) in estimating power requirements. Other antenna combinations must be investigated before a decision is made as to what will constitute the most efficient lunar communication system.

Now to estimate the required power supplied to the input terminals of the transmitter, we need only to designate the type of communication service desired, thus specifying $R$ and $B$. For example with standard broadcast service and a bandwidth of 10 $\mathrm{kc} / \mathrm{s}, R$ is given the value $39 \mathrm{db}$ [C.C.I.R., 1951] and $B=40 \mathrm{db}$ so that $79 \mathrm{db}$ should be added to the curves of figure 16 to obtain the required power in decibels above 1 watt. Thus the power required for this type of service at a range of $10 \mathrm{~km}$ and for a frequency of $300 \mathrm{kc} / \mathrm{s}$ would be about $10 \mathrm{db}$ or $10 \mathrm{w}$. For a lowgrade voice communication service and $6 \mathrm{kc} / \mathrm{s}$ bandwidth, $R=9 \mathrm{db}$ [C.C.I.R., 1951], $B=38 \mathrm{db}$, and 47 $\mathrm{db}$ would be added to the curves. The required power in this case at a distance of $100 \mathrm{~km}$ and for a frequency of $100 \mathrm{kc} / \mathrm{s}$ would be about $16 \mathrm{w}$. 


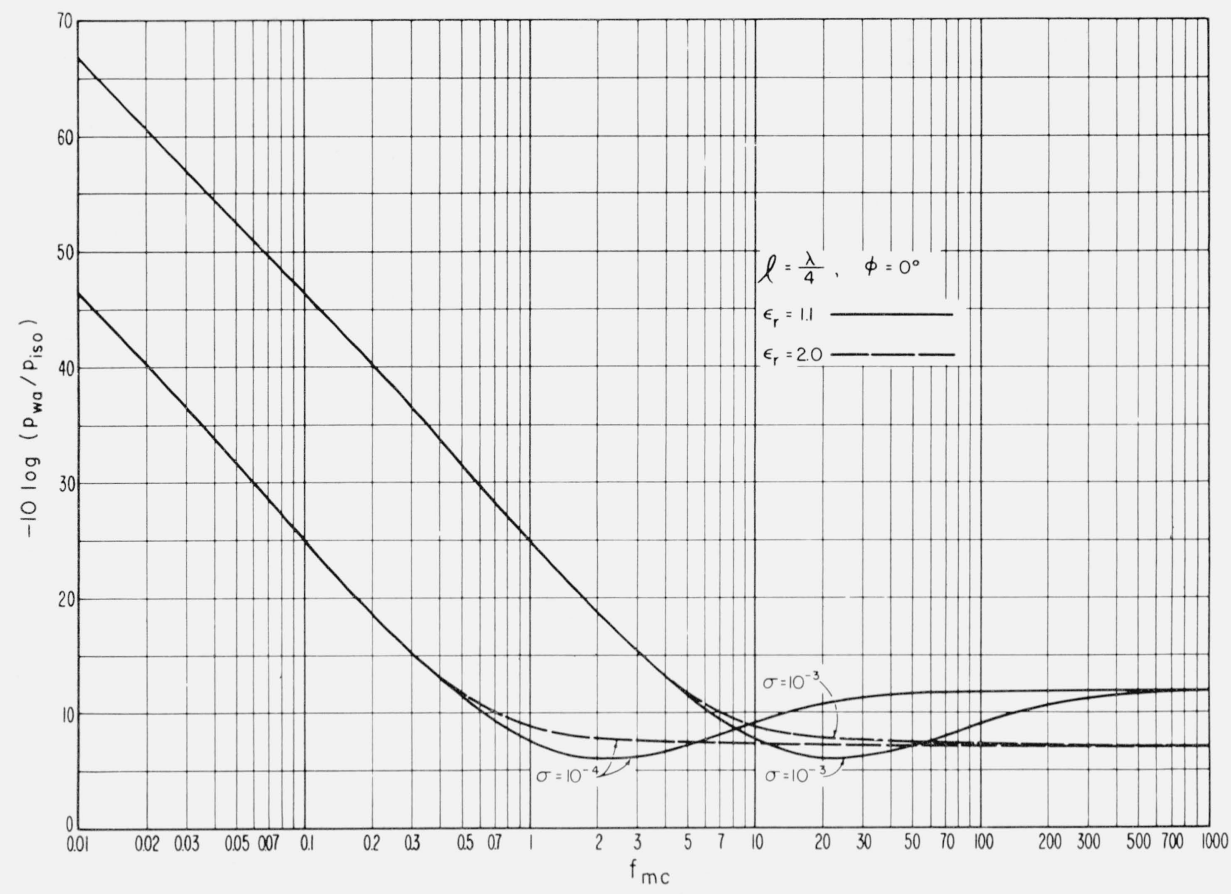

Figure 15. Power loss for horizontal traveling wave antenna lying on the ground.

In the particular idealized system described by figure 16 a number of points should be noted: (1) The required power does not vary appreciably over the range of $\epsilon_{r}$ assumed, and thus for this model the relative permittivity of the lunar surface is not especially critical for propagation considerations; (2) propagation out to somewhat beyond $100 \mathrm{~km}$ is practical for most types of service, at least at MF or below; (3) the curves indicate an optimum frequency exists, depending on the conductivity of the lunar surface and the range of propagation; for the conductivities and distances shown, the optimum frequency lies in the LF band. It should be kept in mind that the effective noise figure of the receiving system was considered to be only a function of galactic noise (which, of course, is extrapolated at the lower frequencies) and a rather low receiver noise figure. If the receiving antenna loss $l_{c}$ were large enough, it is apparent from (19) that $F$ would have higher values than those assumed. Also, during the lunar day, the antenna temperature $t_{c}$ would increase thus making the effective noise figure even higher.

\section{Future Studies}

On the question of further studies concerning communication on the moon, consideration certainly should be given to the following:

1. An attempt should be made to evaluate more precisely the range of values of lunar electromagnetic constants, and especially the electric conductivity; maximum range, optimum frequency, and type of antenna are all dependent on the latter parameter.

2. It is possible that the optimum frequencies indicated in figure 16 are mathematically "true" but not physically real; i.e., that the model and the assumptions made are too far from reality. Earthbased experiments in areas simulating ground conditions on the moon could be undertaken to check their validity.

3. Combinations of antennas other than that of section 6 should be investigated both experimentally and theoretically.

4. Data on galactic noise at low frequencies and not influenced by the earth's ionosphere should be obtained in order to check the validity of (20) and figure 13. Special consideration should be given to possible low frequency solar noise and its effect on lunar "daytime" communication.

5. An investigation of propagation effects assuming a lunar model composed of layered materials and including an ionosphere should be undertaken. Theoretical studies of these two aspects of the problem are now in progress at the Central Radio Propagation Laboratory in Boulder, Colorado. The results of this work, which is sponsored by the Jet Propulsion Laboratory, will be published as a second part to the present report.

The author gratefully acknowledges the assistance of the following personnel in the preparation of this report: J. E. Herman, J. L. Noble, P. G. Ratcliffe, and R. E. Wilkerson. Special thanks go to Mr. Noble for his assistance in the calculation and plotting of the attenuation curves. The author also thanks K. A. Norton and J. R. Wait of the NBS Boulder Laboratories and Paul S. Goodwin of Jet Propulsion Laboratory for their suggestions and guidance. 


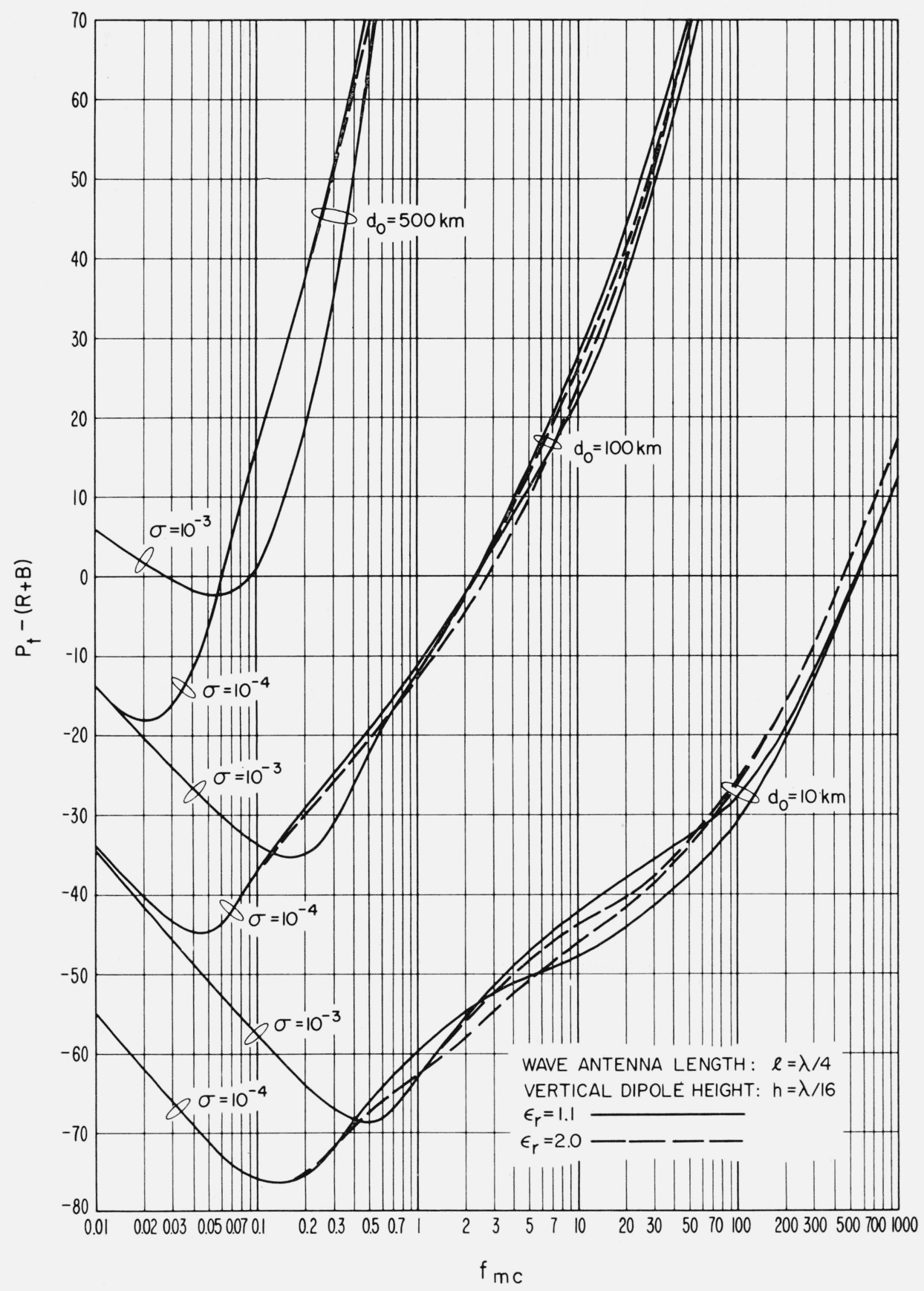

Figure 16. $\mathrm{P}_{\mathrm{t}}-(\mathrm{R}+\mathrm{B})$ for wave antenna and vertical dipole.

\section{Appendix}

\subsection{The Residue Series}

The residue series for radio waves diffracted around a smooth homogeneous sphere as formulated by Bremmer $[1949]^{3}$ is

$$
a_{t}=\sqrt{2 \pi \chi}\left|\sum_{s=0}^{\infty} \frac{\exp \left(i \tau_{s} \chi\right)}{2 \tau_{s}-1 / \delta^{2}}\right|
$$

3 Notice that Bremmer uses egs units whereas the units used in this paper are $\mathrm{mks}$ where $a_{t}$ denotes the attenuation of the field relative to a free space inverse distance field, $\tau_{s}$ and $\delta$ are parameters which depend on the polarization of the wave and the electromagnetic ground constants characterizing the sphere, and $\chi$ is defined by

$$
\chi=\left(2 \pi r_{0} / \lambda\right)^{\frac{2}{3}}\left(d_{0} / r_{0}\right)
$$

with $\lambda$ the free space wavelength, $r_{0}$ the radius of the sphere, and $d_{0}$ the arc distance along the sphere. $\tau_{s}$ is defined by Bremmer, using a time dependence of $\exp (-i \omega t)$ as the roots of the equation 


$$
\frac{H_{2 / 3}^{(1)}\left\{(1 / 3)\left(-2 \tau_{s}\right)^{3 / 2}\right\}}{H_{1 / 3}^{(1)}\left\{(1 / 3)\left(-2 \tau_{s}\right)^{3 / 2}\right\}}=\frac{-e^{-i \pi / 3}}{\delta_{e, m} \sqrt{-2 \tau_{s}}}
$$

where $H_{1 / 3}^{(1)}$ and $H_{2 / 3}^{(1)}$ denote Hankel functions of the first kind of orders one-third and two-thirds and $\delta_{e}$ and $\delta_{m}$ are given by

$$
\begin{aligned}
\delta_{e} & =\frac{i\left(k_{2}^{2} / k_{1}^{2}\right)}{\left(k_{1} a\right)^{1 / 3} \sqrt{\left(k_{2}^{2} / k_{1}^{2}\right)-1}}=K_{e} \exp \left[i\left(135^{\circ}-\psi_{e}\right)\right] \\
\delta_{m} & =\left(k_{1}^{2} / k_{2}^{2}\right) \delta_{e}=K_{m} \exp \left[i\left(45^{\circ}+\psi_{m}\right)\right],
\end{aligned}
$$

with

$$
k_{1}^{2}=(2 \pi / \lambda)^{2}, k_{2}^{2}=k_{1}^{2}\left(\boldsymbol{\epsilon}_{r}+i 60 \lambda \sigma\right) .
$$

$\epsilon_{r}$ is the relative dielectric constant of the ground and $\sigma$ the ground conductivity in mhos per meter.

If we now define $s \equiv 60 \lambda \sigma$ and make use of eqs (6), (7), and (8) in section 3 together with $(\mathrm{A} 1-5)$, the parameters of $(\mathrm{A} 1-4)$ may be expressed in terms of the notation used in this paper:

$$
K_{e}=K_{v}, \psi_{e}=b_{v}^{\circ} / 2 \text { (vertical polarization), }
$$

$K_{m}=K_{h}, \psi_{m}=90^{\circ}-b_{h} / 2$ (horizontal polarization).

$(\mathrm{A} 1-7)$

The parameter $\chi$ given by $(\mathrm{A} 1-2)$ is related to $x_{0}^{\prime}$ [see (9)] by

$$
\chi=\left(2 \pi / 0.3 r_{0}^{2}\right)^{\frac{1}{3}} x_{0}^{\prime},
$$

where $r_{0}$ is expressed in kilometers.

\subsection{Wave Antenna Power Gain}

The signal power $p_{w a}$ received by a wave antenna of length $l$ terminated in its characteristic impedance $Z_{0}=R_{0}+i X_{0}$ is given by Martin and Wickizer [1949] as

$$
p_{u a}=\frac{\left|E_{y}\right|^{2}\left|T_{v}\right|^{2}|S|^{2}}{4 \beta^{2}} \cdot \frac{R_{0}}{\left|Z_{0}\right|^{2}}
$$

where $E_{y}$ is the vertical component of the electric field, $S=(\beta l \cos \phi) S^{\prime}, \beta=2 \pi / \lambda$, and $\phi$ is the angle measuring the direction in which the antenna is pointing ( $\phi=0$ in the forward direction). $T_{v}$ and $S^{\prime}$ are defined in (10) and (11) of section 4 . The signal power $p_{\lambda / 4}$ received by a quarter-wave vertical antenna with load resistance equaling its free space radiation resistance is

$$
p_{\lambda / 4}=\frac{\left|E_{y}\right|^{2}}{4 \beta^{2}} \cdot \frac{g_{\lambda / 4}}{120},
$$

where $g_{\lambda / 4}$ is the gain of the quarter-wave antenna above an isotropic antenna. Thus the wave antenna power gain referred to an isotropic antenna (eq (12) of section 4) is defined in this paper as:

$\left(p_{w a} / p_{i s o}\right)=\left(p_{w a} / p_{\lambda / 4}\right) g_{\lambda / 4}=|S|^{2}\left|T_{v}\right|^{2}\left(120 R_{0} /\left|Z_{0}\right|^{2}\right)$,

where $|S|=|\beta l \cos \phi| \cdot\left|S^{\prime}\right|$.

The theoretical determination of $L_{t}-G_{t}$ or $L_{r}-G_{r}$ for a wave antenna in terms of its terminal resistance is quite difficult. However, since these quantities are essentially power ratios measuring the effectiveness of the antenna, an estimate of their magnitude may be obtained from $(\mathrm{A} 2-3)$. Thus in section 6 the assumption is made that

$$
\begin{aligned}
& L_{t}-G_{t}=-10 \log \left(p_{w a} / p_{i s o}\right) . \\
& \text { 9. References }
\end{aligned}
$$

\section{References}

Barsis, A. P., K. A. Norton, P. L. Rice, and P. H. Elder, Performance predictions for single tropospheric communication links and for several links in tandem, NBS Tech. Note 102 (Aug. 1961). See Appendix III.

Beverage, H. H., C. W. Rice, and E. W. Kellogg, The wave antenna, Trans. AIEE 4\%, 215 (1923).

Bremmer, H., Terrestrial radio waves, Elsevier Publishing Co., Amsterdam (1949).

Brown, R. H., and A. C. B. Lovell, The exploration of space by radio, Chapman and Hall, Ltd., London (1957).

Brown, W. E., A lunar and planetary echo theory, J. Geophys. Res. 65, 3087 (1960).

Burrows, C. R., and M. C. Gray, The effect of the earth's curvature on ground-wave propagation, Proc. IRE 29, 16 (1941).

Carson, J. R., Wave propagation in overhead wires with ground return, Bell System Tech. J. 5, 539 (1926).

C.C.I.R., Bandwidths and signal-to-noise ratios in complete systems, VI Plenary Assembly, International Radio Consultative Committee 1, 30, Geneva (1951).

Coleman, B. L., Propagation of electromagnetic disturbances along a thin wire in a horizontally stratified medium, Phil. Mag. 41, 276 (1950).

Crichlow, W. Q., D. F. Smith, R. N. Morton, and W. R. Corliss, Worldwide radio noise levels expected in the frequency band $10 \mathrm{kc}$ to $100 \mathrm{Mc}$, NBS Circ. 557 (Aug. 1955 ).

Daniels, F. B., A theory of radar reflection from the moon and planets, J. Geophys. Res. 66, 1781 (1961).

Friis, H. T., Noise figures of radio receivers, Proc. IRE 32, 419 (1944).

Martin, C. A., and G. S. Wickizer, Study of Beverage wave antenna for use with low-frequency Loran, RCA, Final Engineering Report on Contract W-28-099-ac-315 (1949).

Menzel, D. H., Cosmic noise survey, Harvard College Observatory, Cambridge 38, Mass.

Norton, K. A., Ground-wave field intensity, Proc. IRE 29, 623 (1941).

Norton, K.A., Transmission loss in radio propagation, Proc. IRE 41, 146 (1953).

Norton, K. A., System loss in radio wave propagation, J Research NBS 63D (Radio Prop.), No. 1, 53 (July-Aug., 1959).

Schelkunoff, S. A., and H. T. Friis, Antennas. theory and practice, John Wiley \& Sons, Inc., New York, N.Y. (1952).

Senior, T. B. A., and K. M. Siegel, A theory of radar scattering by the moon, J. Research NBS 64D (Radio Prop.), No. 3, 217 (May-June, 1960).

Sommerfeld, A., and F. Renner, Strahlungsenergie und Erd-absorption bei Dipolantennen, Ann. Phys. 41, 1 (1942).

Wait, J. R., Radiation resistance of a small circular loop in the presence of a conducting ground, J. Appl. Phys. 24, 646 (1953).

Wait, J. R., Radiation from a ground antenna, Can. J. Technol. 32, 1 (1954).

Wait, J. R., and W. J. Surtees, Impedance of a top-loaded antenna of arbitrary length over a circular grounded screen, J. Appl. Phys. 25, 553 (1954).

Wait, J. R., Effect of the ground screen on the field radiated from a monopole, IRE Trans. Ant. Prop. AP-4, 179 (1956).

Wait, J. R., Transmission of power in radio propagation, Electronic and Radio Engineer 36, 146 (1959).

Wait, J. R., The propagation of electromagnetic waves along the earth's surface, a chapter (pp. 243-290) of Electromagnetic Waves, ed. R. E. Langer (University of Wisconsin Press, 1962).

(Paper 67D1-238) 